\title{
A phase II evaluation of nanoparticle, albumin-bound (nab) paclitaxel) in the treatment of recurrent or persistent platinum- resistant ovarian, fallopian tube, or primary peritoneal cancer: A Gynecologic Oncology Group Study
}

\author{
Robert L. Coleman, MD ${ }^{1}$, William E. Brady, MS², D. Scott McMeekin, MD 3 , Peter G. Rose, \\ MD $^{4}$, John T. Soper, MD ${ }^{5}$, Samuel S. Lentz, MD ${ }^{6}$, James S. Hoffman, MD ${ }^{7}$, and Mark S. \\ Shahin, MD ${ }^{8}$ \\ ${ }^{1}$ Department of Gynecologic Oncology, University of Texas, MD Anderson Cancer Center, \\ Houston, TX 77030 \\ ${ }^{2}$ Gynecologic Oncology Group Statistical \& Data Center; Roswell Park Cancer Institute; Buffalo, \\ NY 14263 \\ ${ }^{3}$ Division of Gynecologic Oncology, University of Oklahoma, Oklahoma City, OK 73190 \\ 4 Division of Gynecologic Oncology; Cleveland Clinic Foundation; Case Western Reserve \\ University; Cleveland, $\mathrm{OH} 44195$ \\ ${ }^{5}$ Division of Gynecologic Oncology; University of North Carolina, Chapel Hill NC 27599 \\ ${ }^{6}$ Division of Gynecologic Oncology; Wake Forest University; Winston-Salem, NC 27157 \\ 7 The Hospital of Central Connecticut Gynecologic Oncology; New Britain, CT 06050 \\ 8 Division of Gynecologic Oncology; Abington Memorial Hospital; Abington, PA 19001
}

\section{Abstract}

Background-Nab-paclitaxel is a novel Cremophor ${ }^{\circledR}$-free nanoparticle of albumin-stabilized paclitaxel, which has favorable efficacy and toxicity characteristics relative to other solvent-based taxanes, such as paclitaxel and docetaxel.

\begin{abstract}
Methods-Eligible patients had platinum- and taxane-resistant ovarian cancer, defined by persistent or progressive disease following primary chemotherapy $(n=5)$ or recurrence within six months of treatment completion $(n=42)$. All patients had measurable disease, no prior therapy for recurrent disease and Gynecologic Oncology Group performance status of $\leq 2$. Treatment was nab-paclitaxel, $100 \mathrm{mg} / \mathrm{m}^{2}$ days $1,8,15$ on a 28 -day schedule. The primary endpoint was Response
\end{abstract}

(C) 2011 Elsevier Inc. All rights reserved.

Corresponding Author: Robert L. Coleman, MD, University of Texas; MD Anderson Cancer Center, Department of Gynecologic Oncology, P.O. Box 301439, Houston, TX 77230, Phone: 713-745-3357, FAX: 713-792-7586, rcoleman@mdanderson.org.

\section{CONFLICT OF INTEREST STATEMENT}

William E. Brady has an Angstrom Pharmaceuticals honorarium of \$50.00. All other co-authors have no conflicts of interest to declare. Robert L. Coleman, MD serves on an National Comprehensive Cancer Network Grants Committee, which has awards funds to support other clinical work with this investigational agent.

Publisher's Disclaimer: This is a PDF file of an unedited manuscript that has been accepted for publication. As a service to our customers we are providing this early version of the manuscript. The manuscript will undergo copyediting, typesetting, and review of the resulting proof before it is published in its final citable form. Please note that during the production process errors may be discovered which could affect the content, and all legal disclaimers that apply to the journal pertain. 
Evaluation Criteria in Solid Tumors v1.0 response rate, evaluated in a 2-stage design (with power of 0.90 for a RR of $25 \%$ and with alpha of 0.05 for RR of $10 \%$ ).

Results-Fifty-one patients were enrolled of which 47 were evaluable; median time from frontline therapy completion to registration was 21 days. Patient demographics include median age: 59 (34-78) years, serous histology: 72\%, and high-grade: $81 \%$. Efficacy: One complete and 10 partial responses were confirmed $(23 \%) ; 17$ patients $(36 \%)$ had stable disease. The median progression-free survival was 4.5 months (95\%CI: 2.2-6.7); overall survival was 17.4 months (95\%CI: 13.2-20.8). Seventeen patients (36\%) had PFS > six months. Toxicity: there were no grade 4 events; grade 3 events were neutropenia (6), anemia (3), GI (2), metabolic (2), pain (2), and leukopenia (1); neurosensory toxicity was observed as grade 2:5, grade 3:1.

Conclusions-Nab-paclitaxel has noteworthy single-agent activity and is tolerable in this cohort of refractory ovarian cancer patients previously treated with paclitaxel.

\section{Keywords}

ovarian cancer; fallopian tube cancer; primary peritoneal cancer; platinum-resistant; taxaneresistant; nab-paclitaxel

\section{INTRODUCTION}

Emergence of drug resistance is responsible for the majority of deaths due to recurrent ovarian cancer. Further complicating this clinical observation is the approximately $25 \%$ of patients in whom platinum and taxane resistance are a phenotype of their primary disease [1]. This has bolstered the continuous search for novel cytotoxic and biologic compounds targeting multiple aspects of the tumor microenvironment.

As a class, the taxanes remain a foundation of primary and recurrent ovarian cancer management strategies. In 1996, paclitaxel was found to result in superior progression free (PFS) and overall (OS) survival in women with suboptimally-cytoreduced ovarian cancer in combination with cisplatin compared to cyclophosphamide + cisplatin [1]. Since this time, the agent has found ubiquitous use in gynecologic malignancies, including recurrent ovarian cancer. Furthermore, other taxane analogues have been described to have clinical activity alone and in combination with other cytotoxics and biological agents in this setting.

The Gynecologic Oncology Group (GOG) has serially investigated several cytotoxic agents in the setting of measurable platinum- and taxane-resistant recurrent ovarian cancer (the GOG 126-queue). To date, 13 compounds have undergone objective evaluation for clinical efficacy in this cohort of women. In these sequentially conducted studies, eligibility criteria and evaluation methodology have remained essentially unaltered, providing a unique historical perspective of efficacy and toxicity, while sparing patient resources. From this investigation, two taxanes, docetaxel and paclitaxel (weekly administration), and pemetrexed have demonstrated sufficient clinical activity to merit further clinical evaluation [2-4]. Herein, we report on the fourth agent, nab-paclitaxel (ABI-007).

Nab-paclitaxel is a novel clinical entity incorporating paclitaxel into an albumin nanoparticle. In this solvent-free formulation, paclitaxel exhibits linear pharmacokinetics, can be administered without hypersensitivity premedication and has been associated in preclinical models with higher intratumoral concentrations of paclitaxel relative to the Cremaphor®-EL (Cr-EL) paclitaxel administration. This latter observation is due to transendothelial cell transport of albumin sinks around tumor [5-7]. Heightened efficacy has been observed in a number of solid tumors, most notably, metastatic breast cancer, where the agent is Food and Drug Administration (FDA) approved. Current administration 
guidelines have suggested that a higher therapeutic ratio can be achieved by administering nab-paclitaxel weekly as opposed to its bolus infusion [6-9]. Thus, given the previous documented clinical efficacy of weekly paclitaxel in patients with recurrent, platinum- and taxane-resistant ovarian cancer, we sought to investigate the efficacy of weekly nabpaclitaxel in this setting.

\section{METHODS}

\section{Patients}

Consistent with other agents evaluated in the GOG 126-series, patients were required to have recurrent or persistent epithelial ovarian, primary peritoneal or fallopian tube carcinoma. All patients were required to be considered platinum- and taxane-resistant or refractory according to standard GOG criteria, i.e., have had a treatment-free interval following platinum/taxane therapy of less than 6 months, have persistent disease at the completion of primary platinum- and taxane-based therapy or have progressed during this therapy. Histological confirmation of the original primary tumor by review of the pathology reports was required. All patients were required to have measurable disease, defined as at least one lesion that could be accurately measured in at least one dimension (longest dimension to be recorded). Each lesion must have been $\geq 20 \mathrm{~mm}$ when measured by conventional techniques, including palpation, plain x-ray, CT, and MRI, or $\geq 10 \mathrm{~mm}$ when measured by spiral CT. Patients were required to have at least one "target lesion" to be used to assess response as defined by Response Evaluation Criteria in Solid Tumors (RECIST) (version 1.0). Patients were to have a GOG Performance Status of 0, 1, or 2; have recovered from effects of recent surgery, radiotherapy, or chemotherapy; and be free of active infection requiring antibiotics. Any hormonal therapy directed at the malignant tumor was to be discontinued at least one week prior to registration; however, continuation of hormone replacement therapy was permitted. Any other prior therapy directed at the malignant tumor, including biological, immunologic agents or radiation therapy, was to be discontinued at least three weeks prior to registration. Allowable initial treatment included high-dose therapy, consolidation, or extended therapy administered after surgical or non-surgical assessment. However, patients were not allowed to receive any additional cytotoxic chemotherapy for management of recurrent or persistent disease, including retreatment with initial chemotherapy regimens. Patients are allowed to receive, but are not required to receive, one additional non-cytotoxic regimen for management of recurrent or persistent disease. Patients were required to have adequate bone marrow function and blood chemistries.

Patients were considered ineligible if they had been previously exposed to nab-paclitaxel; received radiation to more than $25 \%$ of marrow-bearing areas; had uncontrolled hypertension, uncompensated congestive heart failure, symptomatic coronary artery disease, or myocardial infarction within six months. Patients with a history of other invasive malignancies, with the exception of non-melanoma skin cancer, were excluded if there was any evidence of another malignancy within the last 5 years. Finally, all patients were required to provide a written informed consent consistent with all federal, state and institutional investigational requirements prior to receiving protocol therapy.

\section{Treatment Plan}

Eligible patients enrolled onto this Phase II study received nab-paclitaxel $100 \mathrm{mg} / \mathrm{m}^{2}$, intravenously over 30 minutes every seven days on days 1,8 , and 15 of a 28-day cycle. No premedication for the prevention of hypersensitivity reaction (HSR), nausea or vomiting was required but could be administered based on emerging or apparent clinical symptomatology. The maximal body surface area used for dose calculations was $2.0 \mathrm{~m}^{2}$. Patients were 
allowed to continue on therapy indefinitely until: (1) withdrawal of consent, (2) evidence of disease progression or (3) significant side effects precluding further administration, (4) inability to tolerate the lowest doses due to toxicity.

\section{Treatment Modifications}

Hematologic toxicity-Initial treatment modifications consisted of cycle delay and/or dose reduction depending on the severity and duration of the individual toxicity or toxicities attributable to the investigational agent. The use of hematopoietic cytokines and protective reagents [e.g. filgrastim (G-CSF), sargramostim (GM-CSF), and pegfilgrastim] was restricted to those patients who experienced recurrent neutropenic complications despite dosing and delay modifications. Treatment modifications were based on the absolute neutrophil count (ANC) rather than the total white cell count (WBC). Subsequent cycles of therapy were not allowed to be infused until the ANC was greater than or equal to 1500 cells/mcl (CTCAE v3.0 grade 1) and the platelet count was $\geq 100,000 / \mathrm{mcl}$. Therapy could be delayed for a maximum of 2 weeks in response. However, patients who failed to recover adequate counts after a two-week delay will be removed from study. Patients experiencing a first occurrence of febrile neutropenia, and/or documented grade 4 neutropenia persisting $\geq$ 7 days, underwent a one dose level reduction (dose level -1: $80 \mathrm{mg} / \mathrm{m}^{2}$, dose level -2: $60 \mathrm{mg}$ / $\mathrm{m}^{2}$ ) in nab-paclitaxel dosage for subsequent cycles. For recurrent febrile neutropenia, and/or recurrent documented grade 4 neutropenia persisting $\geq 7$ days (after initial dose reduction), prophylactic growth factors were to be administered. Patients with grade 4 thrombocytopenia were also to undergo a 1 level dose reduction.

Non-hematologic toxicity-Grade 2 (or greater) peripheral neuropathy required a reduction of 1 dose level (to $80 \mathrm{mg} / \mathrm{m}^{2}$ ) and delay in subsequent therapy for a maximum of 2 weeks until recovered to grade 1 . This modification was also required for a grade 2 (or greater) renal toxicity. Grade 3 (or greater) elevations in SGOT (AST), SGPT (ALT), alkaline phosphatase or bilirubin required a reduction of 1 dose level and delay in subsequent therapy for a maximum of 2 weeks until recovered to grade 1 . Non-hematologic toxicities with an impact on organ function of grade 2 (or greater) required a reduction of 1 dose level and delay in subsequent therapy for a maximum of 2 weeks until recovered to grade 1, or pre-therapy baseline. While routine premedication to prevent hypersensitivity, nausea, or vomiting was not required, hypersensitivity reactions have been observed in some patients treated nab-paclitaxel. Patients experiencing a severe hypersensitivity reaction, immediate termination of the infusion and administration injectable steroids and epinephrine was recommended, as well as customary patients supportive care. Patients with grade 3 hypersensitivity reactions were allowed to undergo retreatment after standard paclitaxel premedication at the discretion of the investigator. No dose escalations or re-escalations were allowed on this study and intolerance of level -2 dosing $\left(60 \mathrm{mg} / \mathrm{m}^{2}\right)$ necessitated removal from study.

\section{Study parameters and Method of Evaluation}

Following registration but before first infusion, patients were to undergo a complete history and physical exam, including a bimanual pelvic evaluation, clinical tumor assessment, preexisting toxicity assessment, routine hematological and blood chemistries, chest imaging (chest radiograph or computed tomography of the thorax), radiograph tumor measurement (for RECIST 1.0 "target lesion(s)"), serum CA125 and, where appropriate, a serum pregnancy test. A CBC and differential were obtained weekly on therapy, with other laboratory blood testing (similar to baseline) obtained prior to each treatment cycle ( 28 days). 
Standard GOG-RECIST (v1.0) criteria were utilized to evaluate response and progression on this trial. All documented responses were required to undergo confirmation by imaging and/ or exam no sooner than 4 weeks following the initial documentation of response. PFS was calculated from study entry until documented disease progression, death or date of last contact. OS was defined from the date of study entry until death or date of last contact.

\section{Statistical Considerations}

The primary endpoint of this study was frequency of objective tumor response. Secondary endpoints included frequency and severity of adverse effects, and duration of PFS and OS. The study used a flexible, 2-stage accrual design that allowed stopping early for lack treatment activity [8]. During the first stage of accrual, 19 to 26 patients were to be entered and evaluated; if $>=3$ of $19-25$ or $<=42$ of 26 patients had a complete (CR) or partial response (PR), a second phase of accrual was to be initiated, with a total 2-stage accrual of 44 to 51 patients. The regimen would be considered active if at $>=7$ of $44-45$ or $>=8$ of $46-$ 51 patients had a PR or CR. If the true response rate were $10 \%$, the average probability of designating the treatment as active would be limited to $10 \%$; on the other hand, if the true response rate were $25 \%$, the probability of correctly classifying the treatment as active would be $90 \%$.

\section{RESULTS}

A total of 51 patients were enrolled onto this multi-institution cooperative group clinical trial. Three patients were ineligible, due to improper pre-protocol treatment (1), patient not platinum resistant (1), and wrong stage (1). In addition, 1 patient never received study agent. Table 1 presents the characteristics of the eligible and treated patients. While all of the patients on this study were platinum- and taxane-resistant by definition (time from primary treatment completion to first recurrence less than 6 months), a substantial proportion ( $\mathrm{n}=$ $33,70 \%$ ) recurred within 3 months of primary treatment completion, including 6 patients, or $13 \%$, who had increasing disease while on primary platinum and taxane chemotherapy. The median treatment-free interval for the treatment cohort was just 21 days (excluding 5 patients who had persistent, not recurrent, disease. In addition, 12 (26\%) had been previously exposed to bevacizumab.

The median number of cycles administered was 4 (range: 1-40); the most common reason for treatment discontinuation was disease progression occurring in 42 (82\%) patients; treatment-associated toxicity, physician preference and patient preference accounted for 3 $(6 \%), 3(6 \%)$ and $2(4 \%)$ additional patients, respectively.

Table 2 presents the adverse events attributable to the study agent observed in the study. Severe adverse events were uncommon, including myelosuppression, in which no grade 4 toxicities were observed. In addition, severe non-hematological toxicities were infrequently experienced. Neurotoxicity was observed as grade 2 in 5 patients (11\%) and grade 3 in 1 patient (2\%). No grade 3 fatigue was reported in this cohort, however, $15(32 \%)$ reported grade 2 symptoms. Nine patients had a total of 17 treatment delays (each of 7-14 days duration); of the individual instances of delays: 9 were due to protocol-related adverse events; 3 were due to non-protocol illness, and 5 were due to personal reasons or scheduling. Five patients were dose reduced to $80 \mathrm{mg} / \mathrm{m}^{2}$, and one of these was further reduced to 60 $\mathrm{mg} / \mathrm{m}^{2}$.

Of the 47 patients evaluable for efficacy, $11(23 \%, 95 \%$ CI: $12 \%-38 \%)$ were identified with confirmed responses (1 complete response and 10 partial responses); another 17 patients $(36 \%)$ had stable disease. When stratified by a treatment-free interval following primary chemotherapy of $0-3$ and 3-6 months, response rates were: $21 \%$ (7/33) and $29 \%$ 
(4/14), respectively. Of the 12 patients progressing despite carboplatin, paclitaxel and bevacizumab, two (17\%) achieved response. In all, 18 patients (38\%) were progression-free more than 6 months. The median PFS and OS were 4.5 months (95\% CI: 2.2-6.7 months) and 17.4 months (95\% CI: 13.2-20.8 months), respectively, and are presented in Figure 1. At this point, 19 patients were alive, 4 without progression; 23 patients had died as a result of disease progression.

\section{DISCUSSION}

In this multi-institutional phase II, we identified that nab-paclitaxel, administered by weekly infusion is associated with significant clinical activity (objective response: $23 \%$ ) and survival characteristics (PFS: 4.5 months, OS: 17.4 months). These parameters are particularly noteworthy given the treatment population, which was predominately refractory heralded by a median platinum and taxane-free interval of 21 days and $70 \%$ of patients recurring within 3 months of primary treatment completion. In addition, 12 patients enrolling in this trial had progressed despite exposure bevacizumab. Further, despite the short off-treatment interval prior to participation, severe hematological and nonhematological toxicities were infrequently observed, including neurotoxicity.

The investigational agent is a novel nanoparticle formulation of paclitaxel. The impetus to develop this compound was to optimize use of paclitaxel in a Cremophor®-free setting, which could simplify administration by allowing conventional tubing, provide linear pharmacokinetics, and potentially reduce toxicity (e.g. hypersensitivity reactions, neurotoxicity, etc) associated with the solvent $[5,6,8-10]$. In addition, nab-paclitaxel was developed to capitalize on the transendothelial cell transport of albumin which is mediated by the glycoprotein gp60 receptor and caveolar transport. Albumin binds to gp60 and activates CAV1 which induces caveoli formation [11]. Albumin and other plasma constituents migrate across the endothelial cell membrane to the interstitial space. The relevance of this mechanism in cancer therapy stems from the clustering of albumin in tumors by SPARC (secreted protein acidic and rich in cysteine or osteonectin), which binds albumin with high affinity and is overexpressed in many tumor types [12]. Mechanistically this effect has been associated with higher intra-tumoral concentrations of paclitaxel when delivered as nab-paclitaxel relative to Cr-EL paclitaxel. In addition, free paclitaxel in serum following nab-delivery was 5.4 fold reduced compared to tumor concentrations and 2.2 fold lower in normal tissues, accounting for its higher therapeutic ratio relative to $\mathrm{Cr}-\mathrm{EL}$ paclitaxel [13].

Nab-paclitaxel has been previously studied in platinum-sensitive recurrent ovarian cancer patients both as a single agent and in combination with carboplatin. In the single-agent phase II study, 44 evaluable patients (measurable and CA125 assessable) were treated with nabpaclitaxel $260 \mathrm{mg} / \mathrm{m}^{2}$ intravenously over 30 minutes every 21 days for 6 cycles or progression [14]. Prior taxane exposure was $89 \%$, and $92 \%$ of patients had a platinum-free interval of greater than 12 months. Overall RR was 64\%, including 15 patients (34\%) with a complete response. Median PFS was 8.5 months. Severe adverse events were infrequent despite the dose, including $11 \%$ grade 4 neutropenia, $2 \%$ grade 3 fatigue and $13 \%$ grade $2-3$ neuropathy. No HSRs were recorded. In a second phase II study, recently reported in abstract form, nab-paclitaxel $\left(100 \mathrm{mg} / \mathrm{m}^{2}\right.$, days $\left.1,8,15\right)$ was administered in combination with carboplatin (AUC 5, day 1$)$ on a 28-day schedule [15]. All evaluable patients $(\mathrm{n}=38)$ were previously exposed to taxane/platinum therapy (median treatment-free interval: 13 months), had measurable recurrent disease and were treated for at least 6 cycles or until progression. Overall RR was $78 \%$, equally divided as complete and partial responses. The median PFS and OS were 13 months and 30 months, respectively. The most common 
toxicities reported were grade 3-4 neutropenia (43\%), fatigue (33\%) and hypersensitivity to carboplatin (18\%).

Prior to this study's initiation, no prior reports of nab-paclitaxel in either infusion schedule were available in women with platinum- and taxane-resistant ovarian cancer. However, clinical efficacy of nab-paclitaxel administered weekly, appears to be consistent with the clinical efficacy of its parent compound in this setting. Weekly infusions of paclitaxel have been associated with impressive clinical activity in both the primary and recurrent settings, including patients with platinum and taxane-resistant disease [3, 16-21]. Previously, the GOG has evaluated the clinical efficacy of weekly paclitaxel in this research queue (GOG 126-N). In that study of 48 platinum- and taxane-resistant ovarian cancer patients, 10 (21\%) were identified with a clinical response, including 2 complete responders [3]. The median PFS and OS were not recorded in this publication, but were provided in a subsequent report relating response and clinical benefit rates to PFS and OS [22]. In this report, the PFS and OS for weekly paclitaxel were reported as 4.9 and 13.1 months, respectively. In addition, a second multi-center trial of women with recurrent platinum- and taxane-resistant disease required enrollment within 3 months of completion of primary therapy or re-exposure to bolus infusion paclitaxel [20]. Among 36 measurable disease patients, 9 (25\%) experienced a response; PFS and OS for this study's entire cohort of measurable and CA125 evaluable (n = 15) patients was 5.5 months and 13.3 months, respectively.

In the absence of a randomized trial, it is difficult to compare the side effect profile of weekly paclitaxel and weekly nab-paclitaxel; however, some notable observations can be summarized. The infusion of nab-paclitaxel does not require premedication for the prevention hypersensitivity reactions. As such, no HSRs were observed in our treatment cohort. Secondly, while all taxane preparations have been associated with neurotoxicity, it is clear the weekly schedule can significantly reduce its prevalence. In the current report, grade 3 neurotoxicity occurred in 1 patient (2\%). This compares favorably with previously reported rates of grade 3 to 4 neurotoxicity among patients treated with weekly paclitaxel administered at lower doses, which have ranged from $4 \%$ to $11 \%[3,17,20,23,24]$.

Hypothesized mechanisms for clinical efficacy related to infusion schedule include more frequent exposure to cell cycle specific cytotoxicity, dose intensity, endothelial cell apoptosis and leveraged anti-angiogenesis. The mechanisms responsible for an antiangiogenesis effect are varied and include induction of thrombospondin-I, a known inhibitor of VEGF ligand:receptor interaction, modulation of VEGF secretion, endothelial cell apoptosis, regulation of local cytokines and most recently, the suppression of regulatory $t$ cells ( $\left.\mathrm{T}_{\text {regs }}\right)$ [25-35]. While this effect may be more closely tied to the agent, paclitaxel, than to its schedule, more frequent exposure would take advantage of this mechanism [36]. It would be anticipated that weekly taxane administration in combination with agents targeting mechanisms of angiogenesis would have clinical merit as demonstrated in retrospective clinical series [31, 37, 38].

Collectively, our data support the contention that weekly nab-paclitaxel is associated with significant clinical efficacy in highly resistant ovarian cancer patients, and compared to weekly paclitaxel in this setting, may have a more favorable side-effect profile.

\section{Acknowledgments}

This study was supported by National Cancer Institute grants to the Gynecologic Oncology Group Administrative Office (CA 27469) and the Gynecologic Oncology Group Statistical and Data Center (CA 37517). The following Gynecologic Oncology Group member institution participated in this study: Abington Memorial Hospital, University of Oklahoma, University of North Carolina, Cleveland Clinic Foundation and Case Western Reserve Medical Centers, Wake Forest University, M. D. Anderson CCOP, The Hospital of Central Connecticut, Duke 
University Hospital, State University of New York-Downstate, University of Texas, Medical Branch Galveston, University of Texas, M. D. Anderson, The Ohio State University, Fox Chase Cancer Center, Milton S. Hershey Medical Center, University of Cincinnati Medical Center, Rush-Presbyterian-St. Luke's Medical Center, State University of New York-Stony Brook, Women's and Infant's Medical Center. RLC is supported in part by the Anne Rife Cox Chair in Gynecology.

\section{References}

1. McGuire WP, Hoskins WJ, Brady MF, Kucera PR, Partridge EE, Look KY, et al. Cyclophosphamide and cisplatin compared with paclitaxel and cisplatin in patients with stage III and stage IV ovarian cancer.[comment]. New Eng J Med. 1996; 334:1-6. [PubMed: 7494563]

2. Miller DS, Blessing JA, Krasner CN, Mannel RS, Hanjani P, Pearl ML, et al. Phase II evaluation of pemetrexed in the treatment of recurrent or persistent platinum-resistant ovarian or primary peritoneal carcinoma: a study of the Gynecologic Oncology Group. J Clin Oncol. 2009; 27:268691. [PubMed: 19332726]

3. Markman M, Blessing J, Rubin SC, Connor J, Hanjani P, Waggoner S. Phase II trial of weekly paclitaxel $(80 \mathrm{mg} / \mathrm{m} 2)$ in platinum and paclitaxel-resistant ovarian and primary peritoneal cancers: a Gynecologic Oncology Group study. Gynecol Oncol. 2006; 101:436-40. [PubMed: 16325893]

4. Rose PG, Blessing JA, Ball HG, Hoffman J, Warshal D, DeGeest K, et al. A phase II study of docetaxel in paclitaxel-resistant ovarian and peritoneal carcinoma: a Gynecologic Oncology Group study. Gynecol Oncol. 2003; 88:130-5. [PubMed: 12586591]

5. Hawkins MJ, Soon-Shiong P, Desai N. Protein nanoparticles as drug carriers in clinical medicine. Adv Drug Deliv Rev. 2008; 60:876-85. [PubMed: 18423779]

6. Gradishar WJ. Albumin-bound paclitaxel: a next-generation taxane. Expert Opin Pharmacother. 2006; 7:1041-53. [PubMed: 16722814]

7. Gardner ER, Dahut WL, Scripture CD, Jones J, Aragon-Ching JB, Desai N, et al. Randomized crossover pharmacokinetic study of solvent-based paclitaxel and nab-paclitaxel. Clin Cancer Res. 2008; 14:4200-5. [PubMed: 18594000]

8. Desai NP, Trieu V, Hwang LY, Wu R, Soon-Shiong P, Gradishar WJ. Improved effectiveness of nanoparticle albumin-bound (nab) paclitaxel versus polysorbate-based docetaxel in multiple xenografts as a function of HER2 and SPARC status. Anticancer Drugs. 2008; 19:899-909. [PubMed: 18766004]

9. Henderson IC, Bhatia V. Nab-paclitaxel for breast cancer: a new formulation with an improved safety profile and greater efficacy. Expert Rev Anticancer Ther. 2007; 7:919-43. [PubMed: 17627452]

10. Dranitsaris G, Coleman R, Gradishar W. nab-Paclitaxel weekly or every 3 weeks compared to standard docetaxel as first-line therapy in patients with metastatic breast cancer: an economic analysis of a prospective randomized trial. Breast Cancer Res Treat. 2010; 119:717-24. [PubMed: 19495958]

11. John TA, Vogel SM, Minshall RD, Ridge K, Tiruppathi C, Malik AB. Evidence for the role of alveolar epithelial gp60 in active transalveolar albumin transport in the rat lung. J Physiol. 2001; 533:547-59. [PubMed: 11389211]

12. Desai N, Trieu V, Damascelli B, Soon-Shiong P. SPARC Expression Correlates with Tumor Response to Albumin-Bound Paclitaxel in Head and Neck Cancer Patients. Transl Oncol. 2009; 2:59-64. [PubMed: 19412420]

13. Desai N, Trieu V, Yao Z, Louie L, Ci S, Yang A, et al. Increased antitumor activity, intratumor paclitaxel concentrations, and endothelial cell transport of cremophor-free, albumin-bound paclitaxel, ABI-007, compared with cremophor-based paclitaxel. Clin Cancer Res. 2006; 12:131724. [PubMed: 16489089]

14. Teneriello MG, Tseng PC, Crozier M, Encarnacion C, Hancock K, Messing MJ, et al. Phase II evaluation of nanoparticle albumin-bound paclitaxel in platinum-sensitive patients with recurrent ovarian, peritoneal, or fallopian tube cancer. J Clin Oncol. 2009; 27:1426-31. [PubMed: 19224848] 
15. Benigno BB, Burrell MO, Daugherty P, Hernandez P. A phase II nonrandomized study of nabpaclitaxel plus carboplatin in patients with recurrent platinum-sensitive ovarian or primary peritoneal cancer. J Clin Oncol. 2010; 28 Abstr 5011.

16. Boruta DM 2nd, Fowler WC Jr, Gehrig PA, Boggess JF, Walton LA, Van Le L. Weekly paclitaxel infusion as salvage therapy in ovarian cancer. Cancer Invest. 2003; 21:675-81. [PubMed: 14628424]

17. Ghamande S, Lele S, Marchetti D, Baker T, Odunsi K. Weekly paclitaxel in patients with recurrent or persistent advanced ovarian cancer. Int J Gynecol Cancer. 2003; 13:142-7. [PubMed: 12657114]

18. Kita T, Kikuchi Y, Takano M, Suzuki M, Oowada M, Konno R, et al. The effect of single weekly paclitaxel in heavily pretreated patients with recurrent or persistent advanced ovarian cancer. Gynecol Oncol. 2004; 92:813-8. [PubMed: 14984946]

19. Markman M. Weekly paclitaxel in the management of ovarian cancer. Semin Oncol. 2000; 27:3740. [PubMed: 10952125]

20. Markman M, Hall J, Spitz D, Weiner S, Carson L, Van Le L, et al. Phase II trial of weekly singleagent paclitaxel in platinum/paclitaxel-refractory ovarian cancer. J Clin Oncol. 2002; 20:2365-9. [PubMed: 11981009]

21. Thomas H, Rosenberg P. Role of weekly paclitaxel in the treatment of advanced ovarian cancer. Crit Rev Oncol Hematol. 2002; 44 (Suppl):S43-51. [PubMed: 12505598]

22. Rose PG, Tian C, Bookman MA. Assessment of tumor response as a surrogate endpoint of survival in recurrent/platinum-resistant ovarian carcinoma: a Gynecologic Oncology Group study. Gynecol Oncol. 2010; 117:324-9. [PubMed: 20185168]

23. Byrd L, Thistlethwaite FC, Clamp A, Ton C, Hasan J, Jayson GC. Weekly paclitaxel in the treatment of recurrent ovarian carcinoma. Eur J Gynaecol Oncol. 2007; 28:174-8. [PubMed: 17624081]

24. Kaern J, Baekelandt M, Trope CG. A phase II study of weekly paclitaxel in platinum and paclitaxel-resistant ovarian cancer patients. Eur J Gynaecol Oncol. 2002; 23:383-9. [PubMed: 12440808]

25. Albertsson P, Lennernas B, Norrby K. On metronomic chemotherapy: modulation of angiogenesis mediated by VEGE-A. Acta Oncol. 2006; 45:144-55. [PubMed: 16546859]

26. Belotti D, Vergani V, Drudis T, Borsotti P, Pitelli MR, Viale G, et al. The microtubule-affecting drug paclitaxel has antiangiogenic activity. Clin Cancer Res. 1996; 2:1843-9. [PubMed: 9816139]

27. Chen CA, Ho CM, Chang MC, Sun WZ, Chen YL, Chiang YC, Syu MH, Hsieh CY, Cheng WF. Metronomic chemotherapy enhances antitumor effects of cancer vaccine by depleting regulatory $\mathrm{T}$ lymphocytes and inhibiting tumor angiogenesis. Mol Ther. 2010; 18:1233-43. [PubMed: 20372107]

28. Damber JE, Vallbo C, Albertsson P, Lennernas B, Norrby K. The anti-tumour effect of low-dose continuous chemotherapy may partly be mediated by thrombospondin. Cancer Chemother Pharmacol. 2006; 58:354-60. [PubMed: 16333676]

29. Drevs J, Fakler J, Eisele S, Medinger M, Bing G, Esser N, Marme D, et al. Antiangiogenic potency of various chemotherapeutic drugs for metronomic chemotherapy. Anticancer Res. 2004; 24:1759-63. [PubMed: 15274352]

30. Hata K, Osaki M, Dhar DK, Nakayama K, Fujiwaki R, Ito H, et al. Evaluation of the antiangiogenic effect of Taxol in a human epithelial ovarian carcinoma cell line. Cancer Chemother Pharmacol. 2004; 53:68-74. [PubMed: 14569416]

31. Hu L, Hofmann J, Holash J, Yancopoulos GD, Sood AK, Jaffe RB. Vascular endothelial growth factor trap combined with paclitaxel strikingly inhibits tumor and ascites, prolonging survival in a human ovarian cancer model. Clin Cancer Res. 2005; 11:6966-71. [PubMed: 16203789]

32. Klauber N, Parangi S, Flynn E, Hamel E, D’Amato RJ. Inhibition of angiogenesis and breast cancer in mice by the microtubule inhibitors 2-methoxyestradiol and taxol. Cancer Res. 1997; 57:81-6. [PubMed: 8988045]

33. Lau DH, Xue L, Young LJ, Burke PA, Cheung AT. Paclitaxel (Taxol): an inhibitor of angiogenesis in a highly vascularized transgenic breast cancer. Cancer Biother Radiopharm. 1999; 14:31-6. [PubMed: 10850285] 
34. Pasquier E, Honore S, Pourroy B, Jordan MA, Lehmann M, Briand C, et al. Antiangiogenic concentrations of paclitaxel induce an increase in microtubule dynamics in endothelial cells but not in cancer cells. Cancer Res. 2005; 65:2433-40. [PubMed: 15781659]

35. Wang J, Lou P, Lesniewski R, Henkin J. Paclitaxel at ultra low concentrations inhibits angiogenesis without affecting cellular microtubule assembly. Anticancer Drugs. 2003; 14:13-9. [PubMed: 12544254]

36. Lau D, Guo L, Gandara D, Young LJ, Xue L. Is inhibition of cancer angiogenesis and growth by paclitaxel schedule dependent? Anticancer Drugs. 2004; 15:871-5. [PubMed: 15457127]

37. Kamat AA, Kim TJ, Landen CN Jr, Lu C, Han LY, Lin YG, et al. Metronomic chemotherapy enhances the efficacy of antivascular therapy in ovarian cancer. Cancer Res. 2007; 67:281-8. [PubMed: 17210709]

38. Hurt JD, Richardson DL, Seamon LG, Fowler JF, Copeland LJ, Cohn DE, et al. Sustained progression-free survival with weekly paclitaxel and bevacizumab in recurrent ovarian cancer. Gynecol Oncol. 2009; 115:396-400. [PubMed: 19804901] 


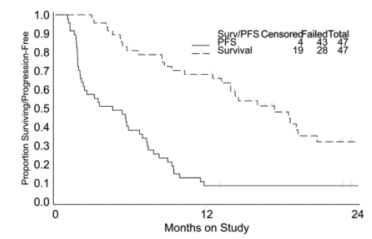

Figure 1.

Progression free (PFS) and overall survival for the study population ( $\mathrm{N}=47$ ) 
Table 1

Characteristics of Evaluable Patients $(\mathrm{N}=47)$

\begin{tabular}{|c|c|c|}
\hline Characteristic & Number of Cases & $\%$ \\
\hline \multicolumn{3}{|l|}{ Age } \\
\hline $30-39$ & 1 & 2.1 \\
\hline $40-49$ & 8 & 17.0 \\
\hline $50-59$ & 16 & 34.0 \\
\hline $60-69$ & 15 & 31.9 \\
\hline $70-79$ & 7 & 14.9 \\
\hline \multicolumn{3}{|l|}{ Race } \\
\hline African American & 9 & 19.1 \\
\hline Hispanic & 2 & 4.3 \\
\hline American Indian & 1 & 2.1 \\
\hline White & 35 & 74.5 \\
\hline \multicolumn{3}{|l|}{ Performance Status } \\
\hline 0 & 31 & 66.0 \\
\hline 1 & 15 & 31.9 \\
\hline 2 & 1 & 2.1 \\
\hline \multicolumn{3}{|l|}{ Site of Disease } \\
\hline Ovary & 41 & 87.2 \\
\hline Fallopian tube & 1 & 2.1 \\
\hline Other & 5 & 10.6 \\
\hline \multicolumn{3}{|l|}{ Cell Type } \\
\hline Adenocarcinoma with Squamous Differentiation & 1 & 2.1 \\
\hline Adenocarcinoma, unspecified & 4 & 8.5 \\
\hline Clear Cell Carcinoma & 4 & 8.5 \\
\hline Endometrioid Adenocarcinoma & 1 & 2.1 \\
\hline Mucinous Adenocarcinoma & 1 & 2.1 \\
\hline Mixed Epithelial Carcinoma & 1 & 2.1 \\
\hline Adenosquamous & 1 & 2.1 \\
\hline Serous Adenocarcinoma & 34 & 72.3 \\
\hline \multicolumn{3}{|l|}{ Grade } \\
\hline 1 & 7 & 14.9 \\
\hline 2 & 2 & 4.3 \\
\hline 3 & 38 & 80.9 \\
\hline \multicolumn{3}{|l|}{ Prior Chemotherapy } \\
\hline 1 Regimen & 47 & 100.0 \\
\hline No Prior Radiation & 47 & 100.0 \\
\hline \multicolumn{3}{|l|}{ Prior Immunotherapy } \\
\hline No & 45 & 95.7 \\
\hline Yes & 2 & 4.3 \\
\hline
\end{tabular}

Prior Hormonal Therapy 


\begin{tabular}{lrr}
\hline Characteristic & Number of Cases & $\%$ \\
No & 45 & 95.7 \\
Yes & 2 & 4.3 \\
Prior Surgery & 47 & 100.0 \\
Cycles of Treatment & & \\
1 & 2 & 4.3 \\
2 & 16 & 34.0 \\
3 & 4 & 8.5 \\
4 & 4 & 8.5 \\
5 & 1 & 2.1 \\
6 & 6 & 12.8 \\
$>6$ & 14 & 29.8 \\
\hline
\end{tabular}


